\title{
FRAÇÕES DE BORO E ÍNDICES DE DISPONIBILIDADE EM SOLOS DO ESTADO DO CEARÁ(1)
}

\author{
F.F.FERREYRAH. ${ }^{(2)} \&$ F.R.SILVA(3)
}

\begin{abstract}
RESUMO
E m 29 amostras superficiais $(0-20 \mathrm{~cm})$ de solos do estado do Ceará, foram determinadas diferentes frações de boro e estabelecidas relações com algumas propriedades do solo e com o boro solúvel, usado como índice de disponi bi lidade para as plantas. As frações de boro foram analisadas, seguindo-se um esquema de fracionamento seqüencial. Determinaram-se o boro solúvel em água (B-Sol), o boro não especificamente adsorvido (B-NEsAd), o boro especificamente adsorvido (B-EsAd), o boro associado a óxidos de Mn (B-OxMn), o boro associado a óxidos de Fe e Al amorfo (B-OxF eA) e a óxidos de Fe e Al cristalino (B-OxFeC), e o boro residual (B-Res). Também foram determi nados o boro total (B-Total) e 0 solúvel em três extratores: água quente, $\mathrm{HCl} 0,05 \mathrm{~mol} \mathrm{~L}^{-1}$ e manitol 0,05 $\mathrm{mol} \mathrm{L} \mathrm{L}^{-1}+$ $\mathrm{CaCl}_{2}$ 0,01 mol L-1. O teor de boro total nos solos variou de 10,5 a $24,0 \mathrm{mg} \mathrm{kg}^{-1}$ (média de 14,4 $\mathrm{g} \mathrm{kg}^{-1}$ ). As frações B-Sol, B-NE sAd e B-E sAd apresentaram teores entre 0,05 e 0,79 $\mathrm{mg} \mathrm{kg}^{-1}$, representando entre 0,3 a 4,4\% do B-Total. Dentre os óxidos, houve predominância do B-OxFeC (média de 4,4 mg kg-1) com teores entre 1 a 2 vezes superiores aos de B-OxFeA (média de 2,72 $\mathrm{mg} \mathrm{kg}^{-1}$ ). Na maioria dos solos, a fração predominante foi o B-Res (média de $6,22 \mathrm{mg} \mathrm{kg}^{-1}$ ), ocluso em minerais silicatados, com teores que variaram de 19,6 a $70,7 \%$ do B-Total. Os valores de B disponível, nos três extratores, correlacionaram-se de forma altamente significativa entre si e com as frações B-Sol, B-NEsAd e B-EsAd. A matéria orgânica e a argila foram as propriedades que se correlacionaram mel hor com o B-Sol, B-NE sAd e B-E sAd.
\end{abstract}

Termos de indexação: boro disponível, propriedades do solo, micronutriente, fracioanmento.

(1) Trabalho financiado pelo CNPq. Recebido para publicação em julho de 1997 e aprovado em dezembro de 1998.

(2) Professor Titular do Departamento de Ciências do Solo, Centro de Ciências Agrárias, Universidade Federal do Ceará (UFC). Caixa Postal 12.168, CEP 60021-970 Fortaleza (CE). Bolsista do CNPq

(3) Pesquisadora do Departamento de Ciências do Solo, Centro de Ciências Agrárias, UFC. 


\title{
SUMMARY: BORON FRACTIONS AND AVAILABILITY INDICES IN SOILS OF CEARÁ, BRAZIL
}

\begin{abstract}
Different soil boron fractions wereevaluated from 29 superficial $(0-20 \mathrm{~cm})$ soil samples in Ceará, Brazil. Relationships were established between soil fractions and soil properties and soluble boron, which has been used as an index of boron availability to the plants. F ractional boron analyses of thesoils werecarried out following a sequential procedure. The foll lowing determi nations werecarried out: thewater sol ubleboron (B-Sol), thenot specifically adsorbed boron (B-NEsAd), the boron associated to manganese oxi de (B-OxMn), the boron associated to $\mathrm{Fe}$ and $\mathrm{Al}$ amorphous oxides (B-OxFeA), the boron associated to $\mathrm{Fe}$ and $\mathrm{Al}$ crystallineoxides (B-OxF eC) and theresidual boron (B-Res). Thetotal boron (B-Total) was assessed as well as the available boron by means of three extractors: hot water $\left(B-\mathrm{H}_{2} \mathrm{Oq}\right)$, $0.05 \mathrm{~mol} \mathrm{~L}^{-1}$ hydrochloric acid $(\mathrm{B}-\mathrm{HCl})$ and $0.05 \mathrm{~mol} \mathrm{~L}^{-1}$ mannitol $+0.01 \mathrm{~mol} \mathrm{~L}^{-1} \mathrm{CaCl}_{2}$ (B-Man). B-Sol, B-NEsAd and EsAd have exhibited amounts ranging from 0.05 to $0.79 \mathrm{mg} \mathrm{kg}^{-1}$, which represents 0.3 to $4.43 \%$ of the B-Total. Among the oxides there was a predominance of $\mathrm{B}-\mathrm{OxF} e \mathrm{C}$ with fractions 1 to 2 times higher than those of $\mathrm{B}-\mathrm{OxF} \mathrm{AA}$. In all soil samples, theB-Res (ocd uded in silicate minerals) was the predominant fraction, with amounts ranging from 19.6 to $70.7 \%$ of theB-Total. TheavailableB, values removed by the three extractors, were highly correl ated among themselves and with $\mathrm{B}-\mathrm{H}_{2} \mathrm{O}, \mathrm{B}-\mathrm{NE}$ sAd and $B$-EsAd fractions. Clay and organic matter were the properties which best correlated with theB-Sol, B-NEsAd and B-EsAd.
\end{abstract}

Index terms: availableboron, soil properties, micronutrient, fractionation.

\section{INTRODUÇÃO}

O boro pode ser encontrado nos solos sob várias formas, as quais variam em sua disponibilidade para as plantas. SegundoKeren \& Bingham (1985), o boro do solo pode ser separado em três categorias distintas: (a) B nos minerais primários (turmalina); (b) B adsorvido pelos constituintes dos solos (minerais de argila, sesquióxidos, matéria orgânica) e (c) B na solução do solo (como ácido bórico e íons borato). Os níveis de boro na solução do solo são controlados, principalmente, por processos de preci pitação e adsorção. Por outro lado, a adsorção de boro é influenciada pelas propriedades dos solos, tais como: conteúdo de argila e área superficial específica (Biggar \& Fireman, 1960; Hatcher et al., 1967), tipos de minerais de argila (Hingston, 1964; Keren \& Mezuman, 1981), sesquióxidos (Hatcher et al., 1967; Sims \& Bingham, 1968), matéria orgânica (Parks \& White, 1952), dentre outros. Diversos pesquisadores determinam as diferentes formas de boro utilizando extratores específicos (de diferentes capacidades de extração) para cada forma, seguindo esquemas de fracionamento seqüenciais e nãoseqüenciais para el ementos metál icos traços em sol os e sedimentos (Tessier et al., 1979; Harrison et al., 1981; Hou et al., 1994, 1996). Nesses esquemas, comumente são determinadas as frações: B solúvel em água, B não especificamente adsorvido na superfície do solo ou trocável, B especificamente adsorvido ou complexado pela matéria orgânica do solo, B ocluso em óxido de Mn, B associado aos óxidos e hidróxidos de Fe e Al amorfos e cristalinos e o B residual, ou associado aos silicatos do solo.

$\mathrm{Na}$ avaliação do B disponível do solo, são utilizados diversos extratores com diferentes capacidades de extração. Dentre os extratores mais utilizados, destacam-se: água quente sob refluxo (Berger \& Truog, 1939; Bataglia \& Raij, 1990); soluções de $\mathrm{HCl}$ 0,05 mol L-1 (Catani et al., 1970; Ponnamperuma et al., 1981; Correa et al., 1985); Mehlich-3 (J in-Yun et al., 1988); soluções de $\mathrm{CaCl}_{2}$ $0,01 \mathrm{~mol} \mathrm{~L}^{-1}$ (Parker \& Gardner, 1981; J effrey \& McCallurn, 1988; Spouncer et al., 1992); acetato de amônio 1 mol L-1 pH 4,8 (J in-Yun et al., 1988); extrato de saturação (Elseewi \& Elmalky, 1979; Gestring \& Soltanpour, 1984) e soluções complexantes como AB-DTPA e manitol (Gestring \& Soltanpour, 1984, 1987). Admite-se que tais extratores diferenciem-se por extrair quantidades ou proporções diferentes de boro das várias formas ou frações presentes no sol o, as quais estão ou podem tornar-se disponíveis às plantas, daí a importância do conhecimento da contribuição de cada uma dessas frações em relação à disponi bilidade de boro para as plantas.

Este trabalho teve como objetivo estudar a distribuição das diferentes frações de boro em amostras superficiais de diferentes classes de solos do estado do Ceará, bem como a relação dessas frações com o boro solúvel em três extratores, usados como índice dedisponibilidade para as plantas, ecom algumas propriedades dos solos. 


\section{MATE RIAL E MÉTODOS}

Para avaliar as diferentes frações de boro no solo, foram col etadas vinte e nove amostras superficiais $(0-20 \mathrm{~cm})$ em unidades de mapeamento do estado do Ceará, baseadas no Levantamento ExploratórioReconhecimento de Solos do estado do Ceará (J acomine et al., 1973a,b). As amostras utilizadas em cada classe de solo foram: cinco de Areia Quartzosa distrófica (AQd), uma deAluvial eutrófico $(\mathrm{Ae})$, uma de Planossolo (PL), duas de Bruno NãoCálcico(NC), duas de Cambissol o eutrófico (Ce), onze de Podzólico Vermelho-Amarelo (PV), três de Latossol o Vermelho-Amarel o distrófico (LVd) e uma de Litossolo eutrófico (Re).

Após a coleta, as amostras foram secas ao ar, passadas em peneiras de $2 \mathrm{~mm}$ de abertura e submetidas à caracterização física e química, seguindo os métodos adotados pela EMBRAPA (1997). Também foram determinados os teores dos óxidos de $\mathrm{Mn}$, pelo método de Chao (1972), e dos óxidos de $\mathrm{Fe}$ amorfos e cristalinos, pelo método de Chao \& Zhou (1983) (Quadro 1).

As diferentes frações de boro foram extraídas, seguindo-se um procedimento de fracionamento seqüencial, usando-se extratores específicos a cada forma ou fração (J in-Yun et al., 1987), conforme procedimento descrito a seguir:

1. Boro solúvel em água fria (B-Sol): extraído com água na temperatura do laboratório $\left(25 \pm 2^{\circ} \mathrm{C}\right)$, na relação solo-água 1:2 e 24 h de agitação;

2. Boro não especificamente adsorvido ou trocável (B-NE sAd): extraído com $\mathrm{CaCl}_{2}$ 0,02 mol L-1 na relação solo-extrator 1:2 e $24 \mathrm{~h}$ de agitação a $25 \pm 2^{\circ} \mathrm{C}$;

\section{Quadro 1. Atributos físicos e químicos de vinte e nove amostras superficiais $(0-20 \mathrm{~cm})$ de solos do estado do Ceará}

\begin{tabular}{|c|c|c|c|c|c|c|c|c|c|c|}
\hline \multicolumn{2}{|c|}{ Solo } & \multicolumn{9}{|c|}{ Atributo(1) $^{(1)}$} \\
\hline \multirow[t]{2}{*}{ № } & Classe $^{(2)}$ & Argila & Silte & MO & pH & CE & $\mathbf{T}$ & Mn-Ox & Fe-OxA & Fe-OxC \\
\hline & & & $\mathrm{g} \mathrm{kg}^{-1}$ & - & & $\mathrm{dS} \mathrm{m}^{-1}$ & $\mathrm{mmol}_{\mathrm{c}} \mathrm{kg}^{-1}$ & 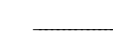 & $-\mathrm{mg} \mathrm{kg}^{-1}$ & - \\
\hline 1 & AQd2 & 84 & 42 & 4,0 & 4,5 & 0,28 & 43 & 0,7 & 187 & 371 \\
\hline 2 & AQd2 & 67 & 44 & 5,7 & 6,1 & 0,20 & 41 & 5,3 & 346 & 911 \\
\hline 3 & AQd4 & 39 & 54 & 3,8 & 6,2 & 0,34 & 31 & 0,8 & 54 & 110 \\
\hline 4 & AQd5 & 53 & 40 & 2,4 & 5,8 & 0,25 & 26 & 0,5 & 44 & 66 \\
\hline 5 & AQd5 & 29 & 17 & 3,4 & 5,5 & 0,21 & 30 & 1,4 & 97 & 413 \\
\hline 6 & Ael & 181 & 468 & 2,2 & 7,0 & 0,58 & 141 & 18,4 & 1.932 & 1.995 \\
\hline 7 & PLI & 66 & 340 & 6,2 & 5,1 & 0,27 & 49 & 1,7 & 816 & 1.188 \\
\hline 8 & NC8 & 80 & 170 & 5,5 & 5,4 & 0,46 & 93 & 19,2 & 2.711 & 2.955 \\
\hline 9 & $\mathrm{NC} 14$ & 109 & 199 & 12,4 & 6,0 & 0,64 & 111 & 131,0 & 1.370 & 3.790 \\
\hline 10 & Cel & 149 & 127 & 31,0 & 7,0 & 0,67 & 94 & 35,7 & 819 & 3.397 \\
\hline 11 & Ce2 & 330 & 112 & 9,0 & 5,9 & 0,80 & 95 & 37,9 & 882 & 4.752 \\
\hline 12 & PVe4 & 201 & 194 & 9,6 & 5,1 & 0,67 & 53 & 19,4 & 2.036 & 3.061 \\
\hline 13 & PVe10 & 199 & 207 & 15,2 & 5,1 & 0,43 & 81 & 2,7 & 2.098 & 3.686 \\
\hline 14 & PVe28 & 160 & 94 & 6,5 & 5,5 & 0,34 & 58 & 91,7 & 804 & 2.678 \\
\hline 15 & PVe32 & 60 & 167 & 9,3 & 6,3 & 0,52 & 63 & 15,6 & 488 & 2.236 \\
\hline 16 & PVe32 & 60 & 42 & 5,7 & 7,0 & 0,76 & 32 & 4,3 & 98 & 493 \\
\hline 17 & PVe40 & 68 & 67 & 3,0 & 6,1 & 0,67 & 28 & 5,7 & 95 & 779 \\
\hline 18 & PV1 & 323 & 154 & 10,0 & 4,6 & 0,18 & 84 & 2,6 & 2.586 & 6.062 \\
\hline 19 & PV2 & 24 & 199 & 3,4 & 4,9 & 0,27 & 22 & 2,3 & 132 & 488 \\
\hline 20 & PV2 & 84 & 179 & 5,0 & 5,5 & 0,59 & 82 & 5,9 & 624 & 1.440 \\
\hline 21 & PV2 & 142 & 89 & 5,7 & 4,9 & 1,16 & 61 & 3,6 & 500 & 568 \\
\hline 22 & PV4 & 85 & 316 & 4,3 & 5,3 & 0,94 & 28 & 13,3 & 2.894 & 4.029 \\
\hline 23 & LVd3 & 475 & 59 & 34,6 & 4,2 & 0,80 & 142 & 1,2 & 2.269 & 4.142 \\
\hline 24 & LVd3 & 468 & 86 & 32,1 & 4,5 & 0,24 & 121 & 1,3 & 1.784 & 3.731 \\
\hline 25 & LVd7 & 130 & 93 & 10,7 & 4,2 & 0,17 & 90 & 1,9 & 1.045 & 2.710 \\
\hline 26 & LVd7 & 136 & 87 & 27,1 & 5,1 & 0,50 & 92 & 11,6 & 902 & 1.150 \\
\hline 27 & Rel & 60 & 160 & 4,1 & 5,9 & 0,70 & 116 & 3,6 & 828 & 1.188 \\
\hline 28 & Re25 & 50 & 253 & 7,0 & 4,7 & 0,19 & 25 & 1,7 & 687 & 884 \\
\hline 29 & Re25 & 60 & 123 & 7,6 & 5,4 & 0,66 & 39 & 2,8 & 707 & 1.395 \\
\hline
\end{tabular}

(1) MO = matéria orgânica; $\mathrm{CE}=$ condutividade elétrica; $\mathrm{T}$ = capacidade de troca de cátions; $\mathrm{Mn}-\mathrm{Ox}=$ teor de $\mathrm{Mn}$ na forma de óxidos; Fe-OxA e Fe-OxC =teor de Fe na forma de óxidos amorfos e cristalinos; respectivamente. ${ }^{(2)}$ Abreviatura da classe de solo seguida de número indica a unidade de mapeamento onde foi coletada a amostra (J acomine et al., 1973a,b). AQd =Areia Quartzosa distrófica; $\mathrm{Ae}=$ Aluvial eutófico; PL = Planossolo; NC = Bruno Não-Cálcico; Ce = Cambissolo; PVe = Podzólico Vermel ho-Amarelo eutrófico; PV =Podzólico Vemel ho-Amarelo; LVd = Latossolo Vermel ho-Amarel o distrófico; Re = Litossolo eutrófico. 
3. Boro especificamenteadsorvido ou compl exado pela matéria orgânica (B-EsAd): extraído com manitol $0,01 \mathrm{~mol} \mathrm{~L}-1+\mathrm{CaCl}_{2} 0,02 \mathrm{~mol} \mathrm{~L}-1$, na relaçãosoloextrator 1:2 e $24 \mathrm{~h}$ de agitação a $25 \pm 2^{\circ} \mathrm{C}$;

4. Boro associado aos óxi dos demanganês (B-OxMn): estimado pela diferença: (B-OxMn +B-OxFeA) B-OxFeA. Após a extração do B-EsAd, em uma subamostra do solo residual, o (B-OxMn + B-OxFeA) foi extraído com oxalato de amônio pH 3,25, na relação solo-extrator 1:5 e agitação no escuro durante $4 \mathrm{~h}$. Numa segunda subamostra, oB-OxF eA foi determinado deacordo com o procedimento indicado na fração 5;

5. B oro associado aos óxidos e hidróxidos de Fee Al amorfos (B-OxFeA): extraído com oxalato de amônio 0,175 mol L-1 pH 3,25, na relação solo-extrator 1:5 e agitação no escuro durante $4 \mathrm{~h}$, após a extração do $\mathrm{B}-\mathrm{O} \times \mathrm{Mn}$ com $\mathrm{NH}_{2} \mathrm{OH}$. $\mathrm{HCl} 0,1 \mathrm{~mol} \mathrm{~L}-1$ em $\mathrm{HNO}_{3} 0,01$ mol L-1, na relação solo-extrator 1:5, e agitação durante $30 \mathrm{~min}$ a $25 \pm 2^{\circ} \mathrm{C}$;

6. Boro associado aos óxidos e hidróxidos deFeeAl cristalinos (B-OxFeC): extraído com oxalato de amônio $0,175 \mathrm{~mol} \mathrm{~L}^{-1} \mathrm{pH} 3,25$, na relação solo:extrator $1: 5$ e 30 min deaquecimento a $100^{\circ} \mathrm{C}$ (Chao \& Zhou, 1983);

7. Boro residual (B-Res): estimado por diferença entre o boro total e a soma das demais frações;

8. Boro total (B-Total): determinado em extratos obtidos após abertura da amostra por fusão, em cadinho de platina, de uma mistura de $0,5 \mathrm{~g}$ de solo com 3,0 g de carbonato de sódio e dissol ução do resíduo em $\mathrm{H}_{2} \mathrm{SO}_{4} 2,0 \mathrm{~mol} \mathrm{~L}-1$, seguindo-se o procedimento indicado por J ackson (1970).

Além do fracionamento, fez-se a extração do boro no solo, utilizando as seguintes sol uções:

1. Água quente $\left(\mathrm{B}-\mathrm{H}_{2} \mathrm{Oq}\right)$ : o boro foi extraído com água quente sob refluxo por $5 \mathrm{~min}$. Usaram-se $20 \mathrm{~g}$ de solo com $40 \mathrm{~mL}$ de água desionizada, depois de frio, adicionaram-setrês gotas de $\mathrm{CaCl}_{2} 0,1 \mathrm{~mol} \mathrm{~L}$ 1 e, em seguida, filtrou-se o material. (Berger \& Truog, 1939; Bataglia \& Raij, 1990);

2. Manitol $0,05 \mathrm{~mol} \mathrm{~L}^{-1}+\mathrm{CaCl}_{2}$ 0,01 mol L-1 (B-Man): utilizaram-se $20 \mathrm{~g}$ de solo com $40 \mathrm{~mL}$ de solução extratora e 8 h de agitação, seguida de filtração (Cartwright et al., 1983), e

3. $\mathrm{HCl} 0,05 \mathrm{~mol} \mathrm{~L}^{-1}(\mathrm{~B}-\mathrm{HCl})$ : usaram-se $10 \mathrm{~g}$ de solo com $20 \mathrm{~mL}$ de solução extratora e $5 \mathrm{~min}$ de agitação, seguida de filtração (Ponnamperuma et al., 1981).

Em todos os extratos, na filtração, utilizou-se papel filtro Whatman 42, e o boro foi determinado pelo método da azometina-H (Wolf, 1971, 1974).

Para todas as frações deboro dosol o foram estabelecidas correlações simples e múltiplas com o boro solúvel $\left(\mathrm{B}-\mathrm{H}_{2} \mathrm{Oq}, \mathrm{B}-\mathrm{M}\right.$ an e $\left.\mathrm{B}-\mathrm{HCl}\right)$, usado comoíndice dedisponibilidade, ecomal gumas propriedades dosolo.

\section{RE SULTADOS E DISCUSSÃO}

De maneira geral, a distribuição das diferentes frações de boro nos solos estudados variou amplamente entre classes de solos e dentro da mesma classe (Quadro 2).

Dentre as frações de boro determinadas, as que apresentaram os menores teores foram: o B-Sol, que representa o boro da sol ução do sol o ( in-Y un et al., 1987), com teores variando de 0,10 a $0,62 \mathrm{mg} \mathrm{kg}^{-1}$, representando de 2,2 a 4,45 \% do boro total; o B-NE sAd ou trocável, extraído pelo $\mathrm{CaCl}_{2}$, com teores entre 0,05 e 0,74 $\mathrm{mg} \mathrm{kg}^{-1}$, representando de 0,32 a $2,27 \%$ do B-Total, e o B-EsAd ou complexado com a matéria orgânica do solo, com teores entre 0,05 e $0,79 \mathrm{mg} \mathrm{kg}^{-1}$, representando de 0,32 a 2,25\% do B-Total. Os conteúdos deB-Sol, com exceção dos solos 23 e 24, foram superiores aos das frações B-NEsAd e B-EsAd, fato também encontrado por J in-Yun et al. (1987), em solos da região costeira plana da Virginia (USA). Esse comportamento deveu-se, possivel mente, ao fato de serem os sol os estudados, em sua maioria, ácidos (Quadro 1). Em condições ácidas, a forma solúvel de boro $\mathrm{H}_{3} \mathrm{BO}_{3}{ }^{\circ}$ predomina sobre a $\mathrm{B}(\mathrm{OH})_{4}{ }^{-}$, sendo sua diferença maior quando aumenta a acidez do meio (Lindsay, 1991). Por outro lado, os íons $\mathrm{B}(\mathrm{OH})_{4}{ }^{-}$podem substituir grupos $\mathrm{OH}$ das bordas dos cristais de argila e serem adsorvidos de forma trocável (Keren \& Mezuman, 1981; Keren et al., 1994). A matéria orgânica, por meio degrupos hidroxila e carboxílicos ácidos, também pode adsorver íons borato de forma trocável ecompl exada como mono-quelatos e bi-quelatos com grupos diol (alcoólicos ou fenólicos) (Yermiyaho et al., 1988).

O boroassodiado aos óxidos do sol o representaram, em média, $64,8 \%$ do B-Total nos solos estudados, o que indica a grande afinidade desses compostos pelo boro. Dentre as frações de boro associadas a óxidos, os menores teores corresponderam ao B-OxMn com teores que variaram de 0,10 a $1,70 \mathrm{mg} \mathrm{kg}^{-1}$, representando de 0,65 a 10,69\% do B-Total. Nesta fração, as maiores percentagens foram encontradas nos sol os Bruno Não-Cálcicos (8 e9) e Cambissol os (10 e 11 ) e os menores nas Areias Quartzosas (1, 3, 4 e 5) que também contêm os maiores e menores teores de óxidos de manganês, respectivamente (Quadro 1).

As frações de boro associadas aos óxidos de Fe e Al amorfos (B-OxFeA) e cristalinos (B-OxFeC), extraíveis pel o oxalato de amônio, variaram de 0,90 a 0,52 $\mathrm{mg} \mathrm{kg}^{-1}$ e 2,2 e 6,7 $\mathrm{mg} \mathrm{kg}^{-1}$, representando de 6,43 a $32,21 \%$ e 18,33 a $46,43 \%$ do B-Total, respectivamente. Os altos conteúdos de boro associados aos óxidos de Fe e Al justificam-se pelo fato de o boro poder adsorver $\mathrm{H}_{3} \mathrm{BO}_{3}{ }^{\circ}$ e íons borato de forma trocável, por simples reações de troca com os gupos hidroxila superficiais; e especificamente adsorvido, formando duas ligações metal-B-O, no interior de complexos que não contêm água entre o íon adsorvido ea superficie do grupo funcional (Sims \& Bingam, 1968; Goldberg, 1993, 1997). 
Quadro 2. Frações de boro e teores de boro solúvel em três extratores de vinte e nove amostras superficiais $(0-20 \mathrm{~cm})$ de solos do estado do Ceará

\begin{tabular}{|c|c|c|c|c|c|c|c|c|c|c|c|}
\hline \multirow{2}{*}{$\begin{array}{l}\text { Número } \\
\text { de solo }\end{array}$} & \multicolumn{7}{|c|}{ Frações de boro' ${ }^{(1)}$} & \multirow{2}{*}{ Boro total } & \multicolumn{3}{|c|}{ Boro solúvel(2) } \\
\hline & Sol & NEsAd & EsAd & OxMn & OxFeA & OxFeC & B residual & & $\mathbf{H}_{2} \mathbf{O}_{q}$ & $\mathrm{HCl}$ & Man \\
\hline & & & & & & $\mathrm{mg} \mathrm{kg}^{-1}$ & & & & & \\
\hline 1 & 0,24 & 0,13 & 0,15 & 0,24 & 1,7 & 2,9 & 5,1 & 10,5 & 0,33 & 0,50 & 0,18 \\
\hline 2 & 0,35 & 0,17 & 0,18 & 0,28 & 3,3 & 4,5 & 5,5 & 14,3 & 0,42 & 0,40 & 0,28 \\
\hline 3 & 0,15 & 0,10 & 0,10 & 0,10 & 0,9 & 2,7 & 9,9 & 14,0 & 0,39 & 0,26 & 0,23 \\
\hline 4 & 0,08 & 0,05 & 0,05 & 0,10 & 2,1 & 4,5 & 8,5 & 15,4 & 0,22 & 0,18 & 0,11 \\
\hline 5 & 0,10 & 0,08 & 0,07 & 0,12 & 2,3 & 4,8 & 6,9 & 14,3 & 0,24 & 0,19 & 0,10 \\
\hline 6 & 0,31 & 0,18 & 0,27 & 0,66 & 1,6 & 2,2 & 6,8 & 12,0 & 0,53 & 0,65 & 0,26 \\
\hline 7 & 0,28 & 0,16 & 0,12 & 0,24 & 2,9 & 5,2 & 2,3 & 11,2 & 0,29 & 0,60 & 0,20 \\
\hline 8 & 0,22 & 0,10 & 0,06 & 0,92 & 3,0 & 4,3 & 3,4 & 12,0 & 0,27 & 0,53 & 0,22 \\
\hline 9 & 0,46 & 0,24 & 0,26 & 1,70 & 3,1 & 3,4 & 6,7 & 15,9 & 0,51 & 0,61 & 0,20 \\
\hline 10 & 0,60 & 0,32 & 0,28 & 1,12 & 2,6 & 4,5 & 7,4 & 16,8 & 1,09 & 1,22 & 0,36 \\
\hline 11 & 0,25 & 0,15 & 0,12 & 0,84 & 2,0 & 4,2 & 6,9 & 14,5 & 0,48 & 0,57 & 0,22 \\
\hline 12 & 0,18 & 0,10 & 0,11 & 0,40 & 1,9 & 3,0 & 5,4 & 11,1 & 0,37 & 0,38 & 0,15 \\
\hline 13 & 0,25 & 0,18 & 0,16 & 0,20 & 2,3 & 3,3 & 4,2 & 10,6 & 0,35 & 0,49 & 0,18 \\
\hline 14 & 0,21 & 0,16 & 0,11 & 1,36 & 4,9 & 6,0 & 3,0 & 15,7 & 0,30 & 0,25 & 0,14 \\
\hline 15 & 0,14 & 0,15 & 0,15 & 0,70 & 2,9 & 3,6 & 2,9 & 10,5 & 0,33 & 0,41 & 0,18 \\
\hline 16 & 0,36 & 0,14 & 0,16 & 0,36 & 2,0 & 3,8 & 6,2 & 13,0 & 0,36 & 0,36 & 0,15 \\
\hline 17 & 0,35 & 0,10 & 0,10 & 0,36 & 2,3 & 3,7 & 4,1 & 11,0 & 0,40 & 0,43 & 0,25 \\
\hline 18 & 0,25 & 0,26 & 0,29 & 0,40 & 2,6 & 4,8 & 6,4 & 15,0 & 0,63 & 0,59 & 0,30 \\
\hline 19 & 0,15 & 0,08 & 0,12 & 0,24 & 2,1 & 3,9 & 6,4 & 13,2 & 0,17 & 0,27 & 0,18 \\
\hline 20 & 0,19 & 0,15 & 0,17 & 0,36 & 2,7 & 3,9 & 4,3 & 11,8 & 0,34 & 0,53 & 0,16 \\
\hline 21 & 0,21 & 0,10 & 0,14 & 0,40 & 3,3 & 4,5 & 11,8 & 20,4 & 0,27 & 0,40 & 0,15 \\
\hline 22 & 0,33 & 0,17 & 0,10 & 0,72 & 2,1 & 5,2 & 15,4 & 24,0 & 0,55 & 0,57 & 0,27 \\
\hline 23 & 0,48 & 0,74 & 0,79 & 0,28 & 5,2 & 6,6 & 5,2 & 19,3 & 0,59 & 0,84 & 0,31 \\
\hline 24 & 0,32 & 0,39 & 0,34 & 0,30 & 4,3 & 5,6 & 6,0 & 17,2 & 0,30 & 0,45 & 0,20 \\
\hline 25 & 0,20 & 0,16 & 0,15 & 0,18 & 2,8 & 5,8 & 5,9 & 15,2 & 0,29 & 0,39 & 0,15 \\
\hline 26 & 0,28 & 0,15 & 0,20 & 0,24 & 4,6 & 6,7 & 8,3 & 20,5 & 0,28 & 0,55 & 0,14 \\
\hline 27 & 0,26 & 0,10 & 0,10 & 0,40 & 2,4 & 4,7 & 3,6 & 11,5 & 0,47 & 0,51 & 0,24 \\
\hline 28 & 0,22 & 0,15 & 0,13 & 0,20 & 2,5 & 5,3 & 5,6 & 14,1 & 0,33 & 0,37 & 0,15 \\
\hline 29 & 0,62 & 0,25 & 0,21 & 0,44 & 2,2 & 3,6 & 6,7 & 14,0 & 0,71 & 0,57 & 0,36 \\
\hline
\end{tabular}

(1) Sol = B solúvel em água; NEsAd = B não especificamente adsorvido; EsAd = B especificamente adsorvido; OxMn =B associado a óxidos de manganês; OxFeA e OxFeC =B associado a óxidos de Feamorfos e cristalinos, respectivamente. (2) índices de B disponível: $\mathrm{H}_{2} \mathrm{O}_{q^{\prime}} \mathrm{HCl}$ e Man = $\mathrm{B}$ extraído pela água quente, $\mathrm{HCl} \mathrm{0,05} \mathrm{mol} \mathrm{L}^{-1}$ e manitol, $0,05 \mathrm{~mol} \mathrm{~L}^{-1}+\mathrm{CaCl}_{2} 0,01$ mol L ${ }^{-1}$, respectivamente.

Os maiores conteúdos de B-OxF eA eB-OxFeC em relação ao B-OxMn são devidos ao fato de serem as quantidades deóxidos deF eeAl nos sol os superiores às dos óxidos de manganês. Entretanto, quando se estabel eceram relações com as médias dos teores de óxidos de $M n$ e de óxidos de Fe amorfos e cristal inos com o boroligado a seus respectivos óxidos, verificousequea relação $\mathrm{Fe}$-OxA/B-OxF eA eF e-OxC/B-OxFeC foi próxima - 115,9 e106,1, respectivamente, enquanto a relação $\mathrm{Mn}$-Ox/B-OxMn foi aproximadamentetrês vezes menor - 31,9. Isto evidencia que a afinidade dos óxidos de manganês pelo boro é superior à dos óxidos de FeeAl.

A fração B-Res, que representa o boro ligado aos minerais silicatados do solo, apresentou teores que variaram de 2,3 a 15,4 $\mathrm{mg} \mathrm{kg}^{-1}$, representando de 19,11 a $70,71 \%$ do boro total do solo. Comparando todas as frações de boro individualmente, o B-Res predominou em 23 dos 29 solos estudados, constituindo, em média, $42,42 \%$ do B-Total. Nos outros seis solos, a fração predominante foi o $\mathrm{B}-\mathrm{OxFeC}$. Entretanto, quando se considerou o boro associado a óxidos minerais (soma), o boro associado a óxidos passou a ser predominante.

A distribuição das frações de boro em relação ao boro total não seguiu um padrão definido nas diferentes classes e nem dentro da mesma classe de solo. Essefatoé expl icado pela ampla faixa devariação dos atributos físi cos e quími cos dos sol os estudados, certamente causada por diferenças no material de origem e nos processos de desenvol vimento do sol o. A influência do material de origem na distribuição relativa das frações de boro, para quatro classes diferentes e para sol os da mesma classe, éilustrada nas figuras 1 e 2, respectivamente. $\mathrm{Na}$ figura 1 , observa-se que nos solos $3(\mathrm{AQd} 4)$ e 11 (Ce), originados de sedimentos do grupo Barreiras e Arenito Calcífero da Formação Jandaíra, 
respectivamente, o B-Res é a fração predominante; enquanto no solo 14 (PVe 28), formado de material areno-argiloso relativo ao terciário que recobre rochas do Pré-Cambriano, e no solo $23\left(\mathrm{LVd}_{3}\right)$, originado de arenitos argilosos da Formação Exu (J acomine et al., 1973a), o B-OxF eA e o B-OxF eC são as frações predominantes. Na figura 2, verificase que o solo 15 (PVe 32), derivado de gnaisse, migmatito, micaxisto e granito, apresenta maior proporçãonas frações B-OxF eC eB-OxF eA do quea fração B-Res; no solo 17 (PVe 40), derivado de sedimentos argilo-arenosos do terciário, as proporções das frações B-OxF eC e B-Res são próximas; enquanto o solo 18 (PV 1), derivado de gnaissee migmatito do Pré-Cambriano, e o 21 (PV 2), derivado de sedimentos do grupo Barreiras, apresentam o B-Res em maior proporção. Deve-se salientar que, além do material de origem, a textura do solo, o pH e a lixiviação também são considerados fatores que influem de forma dominante na distribuição das diversas frações de boro no solo (Shorrocks, 1997).

Os teores de boro solúvel, considerando todas as amostras sem distinção de classes de sol os, variaram de 0,17 a 1,09 $\mathrm{mg} \mathrm{kg}^{-1}$, com média de $0,40 \mathrm{mg} \mathrm{kg}^{-1}$ para a água quente; de 0,18 a $1,22 \mathrm{mg} \mathrm{kg}^{-1}$, com média de $0,49 \mathrm{mg} \mathrm{kg}^{-1}$ para o extrator $\mathrm{HCl}$ 0,05 mol L-1, e de 0,10 a 0,36 $\mathrm{mg} \mathrm{kg}^{-1}$, com média de $0,20 \mathrm{mg} \mathrm{kg}^{-1}$ para o manitol (Quadro 2). O extrator $\mathrm{HCl}$ extraiu mais boro do que a água quente e o manitol (complexante). As maiores quantidades de boro extraídas pel o $\mathrm{HCl}$ 0,05 mol L-1 foram causadas possivel mente pela maior solubilização de minerais quecontinham boro, enquanto as baixas quantidades de boro extraídas pel o manitol são atribuídas ao fato de serem ácidos os solos usados neste estudo. O manitol forma complexos principalmente com íons borato, os quais são predominantes em solos alcalinos (Biswapati \& Kumar, 1993).

Os coeficientes de correl ação simples entre o boro solúvel e as frações de B nos solos (Quadro 3) mostram correlação positiva e significativa a $1 \%$ de probabilidade entre boro extraído pela água quente, $\mathrm{HCl}$ e manitol, quando relacionados entre si, indicando que eles extraíram quantidades proporcionais deborodo sol o. Quando secorrelacionou o boro extraído pel os três extratores com o boro nas diferentes frações do sol o, verificou-se que as frações B-Sol, B-NEsAd eB-EsAd apresentaram coeficientes de correlação altamente significativos, indicando a importante contribuição dessas frações na quantidade de boro solúvel, nos três extratores, usado como índice de disponi bi lidade para as plantas. $\mathrm{O} \mathrm{B}-\mathrm{OxMn}$ correlacionou-se de forma significativa com o $\mathrm{B}-\mathrm{H}_{2} \mathrm{Oq}$ e o $\mathrm{B}-\mathrm{HCl}$ e não-significativa com o B-Man. As outras três frações (B-OxF eA, B-OxF eC e $B$-Res) não apresentaram correlação com boro solúvel. Vê-se, no quadro 3 , que o $\mathrm{B}-\mathrm{H}_{2} \mathrm{Oq}, \mathrm{B}-\mathrm{HCl}$ e $\mathrm{B}-\mathrm{Man}$ representam principalmente o boro das frações B-Sol eB-NEsAd, consideradas prontamente disponíveis para as plantas (fator intensidade); eem menor proporção o das frações B-E SAd e B-OxMn (com exceção do B-Man), consideradas lentamente disponíveis (fator quantidade). As frações B-OxFeA, B-OxF eC e B-Res não influenciaram o boro solúvel, nos três extratores, fato que está de acordo com o indicado por J in-Y un et al. (1987) de que estas frações contribuem com quantidades muito pequenas de boro para plantas de cicl o de desenvol vimento curto. Essa afirmativa é reforçada pelas correlações entre os índices de boro disponível ea soma das frações de B (Quadro 3), quando se observou que a inclusão do B-OxFeA reduziu, de maneira acentuada, os coeficientes de correlação. Considerando que os

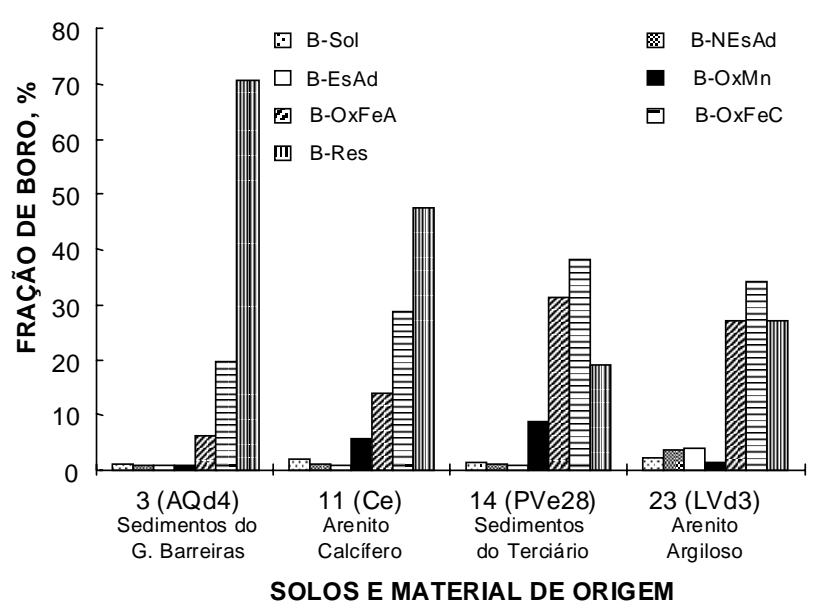

Figura 1. Distribuição percentual das frações de boro em quatro classes de solos de diferentes materiais de origem.

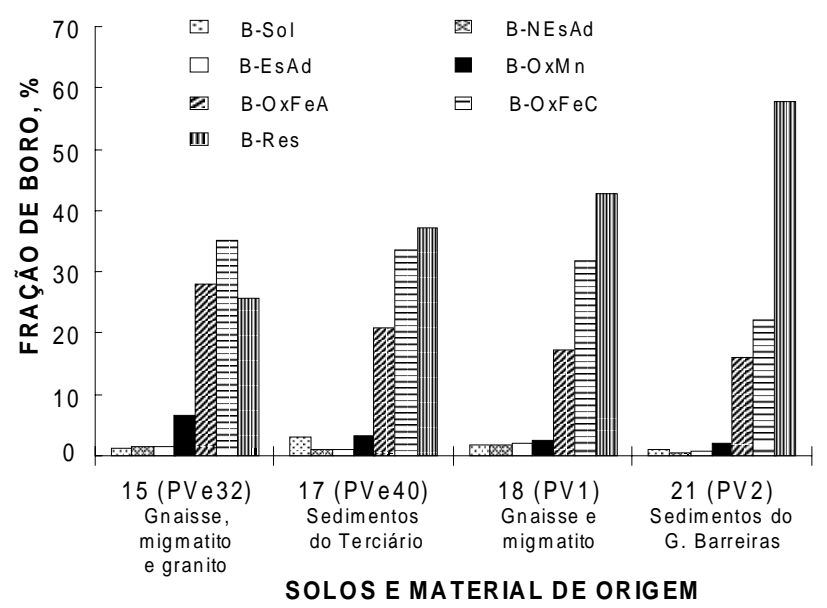

Figura 2. Distribuição percentual de quatro Podzólicos Vermelho-Amarelos de diferentes materiais de origem. 
extratores utilizados constituem índices de disponibilidade de boro razoavelmente bons (Ponnamperuma et al., 1981; Cartwright et al., 1983; Bataglia \& Raij, 1990), embora não tenham sido utilizadas cultura ou planta indicadora, os resultados obtidos no presente trabalho confirmam os encontrados por J in-Yun et al. (1987), com o cultivo de milho, e por Tsadilas et al . (1994), com oliveira e cevada.

O quadro 3 mostra, ainda, as correlações entre as diferentes frações de boro no solo. Nesse quadro, observa-se que o B-Sol correlacionou-se de forma altamente significativa com o B-NEsAd e B-EsAd, significativa com o B-OxMn e não-significativa com o B-OxFeA, B-OxFeC e B-Res. Tais resultados indicam que o conteúdo de boro da sol ução do solo é dependente das frações B-NE sAd, B-E sAd e B-OxM n e, de maneira geral, evidenciam que o equilíbrio químico dessas frações envolve as reações reversíveis indicadas na equação (1); enquanto as demais frações não contribuem ou sua contribuição é inexpressiva em períodos de tempo curto.

$$
\mathrm{B}-\mathrm{Sol} \Leftrightarrow \mathrm{B}-\mathrm{NESAd} \Leftrightarrow \mathrm{B}-\mathrm{ESAd} \Leftrightarrow \mathrm{B}-\mathrm{OxMn}
$$

As correlações das frações B-NEsAd e B-EsAd com o B-OxMn não foram significativas; enquanto com oB-OxF eA foram altamente significativas, fato também encontrado por Tsadilas et al . (1994). A falta de correlação do B-NEsAd eoB-EsAd com oB-OxMn possivelmente foi devida à ampla faixa de variação dos óxidos de manganês nos sol os eaos baixos val ores absolutos de seus teores, quando comparados aos de argila e de matéria orgânica (Quadro 1), responsáveis pela maior parte dos sítios de troca e de adsorsão específica no solo; enquanto as correlações altamente significativas dessas frações com o B-OxFeA devem-se aos óxidos amorfos de Fe e $A \mathrm{I}$, os quais, além de serem encontrados em quantidades apreciáveis no solo, adsorvem boro de forma trocável e especificamente adsorvido. Por outro lado, os al tos val ores de correl ação verificados entre as frações B-NEsAd e B-EsAd ( $\left.r=0,965^{* *}\right)$ e entre as frações B-OxFeA e B-OxFeC ( $\left.r=0,781^{* *}\right)$ indicam que nesses pares as frações de boro são dependentes entre si.

Nas análises de correlação entre as formas de boro e os atributos do solo (Quadro 4), verificou-se que o pH não se correlacionou com o boro solúvel nem com o das frações de boro do solo, exceto com o $\mathrm{B}-\mathrm{OxMn}$ e B-OxFeC, resultados, em sua maioria, contrários aos reportados por Tsadilas et al . (1994). Possivelmente, isso esteja associado ao fato de os sol os usados no estudo serem ácidos (pH entre 4,5 e 7,0 ); enquanto nos solos usados por Tsadilas et al . (1994) o pH variou de 3,9 a 8,5. O aumento do $\mathrm{pH}$ reduz as cargas positivas dependentes do $\mathrm{pH}$, até o ponto de carga zero (PCZ), que podem adsorver íons $\mathrm{B}(\mathrm{HO})_{4}{ }^{-}$por simples mecanismos de troca. Entretanto, a elevação do $\mathrm{pH}$, acima do PCZ, comumenteresulta em aumento da adsorção de boro pelos solos, uma vez que a maioria de seus componentes apresenta uma adsorção máxima de boro a valores depH acima de 7, a qual possivelmente envolve mecanismos de troca de ligantes com os grupos funcionais dihidroxi ehidroxicarboxílicos dos

Quadro 3. Coeficientes de correlação si mples entre o boro solúvel em três extratores e as frações de boro em solos do estado do Ceará

\begin{tabular}{|c|c|c|c|c|c|c|c|c|c|}
\hline \multirow{2}{*}{$\begin{array}{l}\text { Forma de } \\
\text { boro solúvel }\end{array}$} & \multicolumn{9}{|c|}{ Coeficiente de correlação(1) } \\
\hline & $\mathrm{B}-\mathrm{H}_{2} \mathrm{Oq}$ & B-HCl & B-Man & B-Sol & NEsAd & B-E sAd & B-OxMn & B-OxFeA & B-OxFeC \\
\hline $\begin{array}{l}\mathrm{B}-\mathrm{H}_{2} \mathrm{Oq} \\
\mathrm{B}-\mathrm{HCl} \\
\mathrm{B}-\mathrm{Man}\end{array}$ & 1 & $\begin{array}{l}0,829 * * \\
1\end{array}$ & $\begin{array}{l}0,840^{* *} \\
0,711^{* *} \\
1\end{array}$ & $\begin{array}{l}0,789 * * \\
0,746 * * \\
0,770^{* *}\end{array}$ & $\begin{array}{l}0,485^{* *} \\
0,598 * * \\
0,583^{* *}\end{array}$ & $\begin{array}{l}0,429 * \\
0,564^{* *} \\
0,469^{*}\end{array}$ & $\begin{array}{l}0,396^{*} \\
0,379^{*} \\
0,219^{\text {ns }}\end{array}$ & $\begin{array}{r}-0,059^{n s} \\
0,187^{n s} \\
-0,018^{n s}\end{array}$ & $\begin{array}{c}-0,081^{n s} \\
0,088^{n s} \\
-0,089^{n s}\end{array}$ \\
\hline \multicolumn{10}{|c|}{ F ração } \\
\hline $\begin{array}{l}\text { B-Sol } \\
\text { B-NEsAd } \\
\text { B-EsAd } \\
\text { B-OxMn } \\
\text { B-OxFeA } \\
\text { B-OxFeC }\end{array}$ & & & & 1 & $\begin{array}{l}0,602 * * \\
1\end{array}$ & $\begin{array}{l}0,541 * * \\
0,965 * * \\
1\end{array}$ & $\begin{array}{l}0,370^{*} \\
0,105^{\text {ns }} \\
0,042^{\text {ns }} \\
1\end{array}$ & $\begin{array}{l}0,212^{\text {ns }} \\
0,572^{* *} \\
0,535^{* *} \\
0,224^{\text {ns }} \\
1\end{array}$ & $\begin{array}{l}0,051^{\text {ns }} \\
0,402^{*} \\
0,317^{\text {ns }} \\
-0,067^{\text {ns }} \\
0,781^{* *} \\
1\end{array}$ \\
\hline B-Res & $0,166^{\mathrm{ns}}$ & $0,004^{\mathrm{ns}}$ & $0,082^{\mathrm{ns}}$ & $0,051^{\mathrm{ns}}$ & $-0,061^{\mathrm{ns}}$ & $-0,052^{\mathrm{ns}}$ & $-0,041^{\mathrm{ns}}$ & $-0,224^{\mathrm{ns}}$ & $0,025^{\mathrm{ns}}$ \\
\hline B-Total & $0,206^{\text {ns }}$ & $0,206^{\text {ns }}$ & $0,128^{\text {ns }}$ & $0,251^{\mathrm{ns}}$ & $0,366^{*}$ & $0,324^{\mathrm{ns}}$ & $0,142^{\text {ns }}$ & $0,446 *$ & $0,605^{* *}$ \\
\hline$\sum \mathrm{B} 1^{(2)}$ & $0,712^{* *}$ & $0,751^{* *}$ & $0,722 * *$ & & & & & & \\
\hline$\sum \mathrm{B} 2^{(2)}$ & $0,631^{* *}$ & $0,709 * *$ & $0,653^{* *}$ & & & & & & \\
\hline $\bar{\Sigma} \mathrm{B} 3^{(2)}$ & $0,659 * *$ & $0,697 * *$ & $0,554^{* *}$ & & & & & & \\
\hline$\sum \mathrm{B}^{(2)}$ & $0,238^{\text {ns }}$ & $0,432 *$ & $0,223^{\text {ns }}$ & & & & & & \\
\hline
\end{tabular}

(1) *; ** Significativo a 5 e 1\%, respectivamente, ${ }^{\text {ns }}$ não-significativo. ${ }^{(2)} \sum B 1=B-S o l+B-N E s A d ; \sum B 2=B-S o l+B-N E s A d+B-E s A d$; $\Sigma B 3=B-S o l+B-N E s A d+B-E s A d+B-O x M n ; \sum B 4=B-S o l+B-N E s A d+B-E s A d+B-O x M n+B-O x F e A$. 
Quadro 4. Coeficientes de correlação simples e múltiplos entre as formas de boro no solo (variável dependente) e atributos dos solos (variável independente) do estado do Ceará

\begin{tabular}{|c|c|c|c|c|c|c|c|c|c|c|}
\hline \multirow{3}{*}{$\begin{array}{l}\text { Forma de boro } \\
\text { solúvel }{ }^{(3)}\end{array}$} & \multicolumn{10}{|c|}{ Coeficiente de correlação $(r)^{(1)}$} \\
\hline & \multicolumn{6}{|c|}{ Simples } & \multicolumn{4}{|c|}{ Múltiplo(2) } \\
\hline & pH & MO & Argila & Fe-OxA & Fe-OxC & Mn-Ox & $\mathbf{A}$ & B & C & D \\
\hline $\mathrm{B}-\mathrm{H}_{2} \mathrm{Oq}$ & $0,342^{\mathrm{ns}}$ & $0,408 *$ & $0,243^{\mathrm{ns}}$ & $0,269^{\text {ns }}$ & $0,458 *$ & $0,187^{\mathrm{ns}}$ & $0,413^{\mathrm{ns}}$ & $0,484 *$ & $0,484^{\mathrm{ns}}$ & $0,620 *$ \\
\hline $\mathrm{B}-\mathrm{H}^{2} \mathrm{Cl}$ & $0,149^{\text {ns }}$ & $0,611 * *$ & $0,373 *$ & $0,404 *$ & $0,502 * *$ & $0,152^{\text {ns }}$ & $0,617 * *$ & $0,502 *$ & $0,502^{\text {ns }}$ & $0,721 * *$ \\
\hline B-Man & $0,193^{\mathrm{ns}}$ & $0,303^{\text {ns }}$ & $0,259^{\text {ns }}$ & $0,334^{\mathrm{ns}}$ & $0,396 *$ & $-0,024^{\mathrm{ns}}$ & $0,309^{\text {ns }}$ & $0,398^{\mathrm{ns}}$ & $0,432^{\text {ns }}$ & $0,490^{\mathrm{ns}}$ \\
\hline \multicolumn{11}{|c|}{ F ração } \\
\hline B-Sol & $0,202^{\mathrm{ns}}$ & $0,501 * *$ & $0,241^{\mathrm{ns}}$ & $0,201^{\mathrm{ns}}$ & $0,295^{\mathrm{ns}}$ & $0,235^{\mathrm{ns}}$ & $0,526 *$ & $0,300^{\mathrm{ns}}$ & $0,325^{\mathrm{ns}}$ & $0,577^{\mathrm{ns}}$ \\
\hline B-NEsAd & $-0,271^{\mathrm{ns}}$ & $0,791 * *$ & $0,748 * *$ & $0,428 *$ & $0,525 * *$ & $0,050^{\mathrm{ns}}$ & $0,834 * *$ & $0,525^{*}$ & $0,543^{*}$ & $0,837 * *$ \\
\hline B-E sAd & $-0,241^{n s}$ & $0,747 * *$ & $0,725^{* *}$ & $0,375^{*}$ & $0,440 *$ & $0,019^{\text {ns }}$ & $0,797 * *$ & $0,442^{\text {ns }}$ & $0,462^{\text {ns }}$ & $0,807 * *$ \\
\hline $\mathrm{B}-\mathrm{O} \times \mathrm{M} \mathrm{n}$ & $0,371 *$ & $0,102^{\text {ns }}$ & $0,065^{\text {ns }}$ & $0,265^{\mathrm{ns}}$ & $0,464 *$ & $0,905^{* *}$ & $0,103^{\text {ns }}$ & $0,495^{*}$ & $0,922 * *$ & $0,933 * *$ \\
\hline $\mathrm{B}-\mathrm{O} \times \mathrm{F}$ eA & $-0,365^{\mathrm{ns}}$ & $0,623 * *$ & $0,489 * *$ & $0,224^{\mathrm{ns}}$ & $0,279^{\text {ns }}$ & $0,257^{\mathrm{ns}}$ & $0,627 * *$ & $0,279^{\text {ns }}$ & $0,332^{\mathrm{ns}}$ & $0,705^{* *}$ \\
\hline B-OxFeC & $-0,497 *$ & $0,490 * *$ & $0,345^{\text {ns }}$ & $0,159^{\text {ns }}$ & $0,204^{\mathrm{ns}}$ & $-0,039^{\text {ns }}$ & $0,490 *$ & $0,204^{\mathrm{ns}}$ & $0,238^{\mathrm{ns}}$ & $0,496^{\text {ns }}$ \\
\hline B-Res & $0,017^{\mathrm{ns}}$ & $-0,032^{\text {ns }}$ & $-0,036^{\mathrm{ns}}$ & $0,101^{\mathrm{ns}}$ & $0,004^{\mathrm{ns}}$ & $-0,066^{\text {ns }}$ & $0,037^{\text {ns }}$ & $0,163^{\text {ns }}$ & $0,165^{\mathrm{ns}}$ & $0,176^{\text {ns }}$ \\
\hline B-T otal & $-0,228^{\text {ns }}$ & $0,413^{*}$ & $0,304^{\mathrm{ns}}$ & $0,271^{\mathrm{ns}}$ & $0,256^{\text {ns }}$ & $0,121^{\mathrm{ns}}$ & $0,414^{\mathrm{ns}}$ & $0,279^{\text {ns }}$ & $0,288^{\text {ns }}$ & $0,454^{\mathrm{ns}}$ \\
\hline
\end{tabular}

(1) *; ** Significativo a 5 e 1\%, respectivamente, ${ }^{\text {ns }} \mathrm{Não-significativo.}{ }^{(2)}$ Variáveis independentes incluídas na correlação: $\mathrm{A}=\mathrm{MO}$ e argila; $\mathrm{B}=\mathrm{MO}$, argila e Fe-OxA; C = MO, argila, Fe-OxA e Fe-OxC; D = MO, argila, Fe-OxA, Fe-OxC e Mn-Ox.

compponentes do solo (Yermiyaho et al., 1988, Goldberg, 1993, 1997).

A matéria orgânica do solo correlacionou-se de forma altamente significativa com o boro extraído pelo $\mathrm{HCl} 0,05 \mathrm{~mol} \mathrm{~L}^{-1}(r=0,611)$, significativa com a água quente $(r=0,408)$ e não-significativa com o manitol ( $r=0,303$ ) (Quadro 4). O HCl 0,05 mol L-1 apresentou correlação significativa com o teor de óxido de Fe amorfo e altamente significativa com o teor de óxido de Fe cristalino, enquanto a água quente e manitol apresentaram correlação significativa apenas com os óxidos de Fe cristalino.

Nas análises de correlação múltiplas, o extrator $\mathrm{HCl}$ 0,05 mol L-1 apresentou maiores valores de $r$ quando relacionados com a matéria orgânica eargila do solo e quando relacionados com mais de duas propriedades dos solos. A fração B-OxMn esteve diretamente associada aos conteúdos de óxidos de manganês dos solos, apresentando o mais alto coeficiente de correlação simples entre todas as combinações testadas. Por outrolado, as análises de correlaçãosimples emúltiplas também demonstraram que os conteúdos de argila e matéria orgânica dos sol os foram os atributos que mais contribuíram nos conteúdos de boro das frações B-NEsAd e B-EsAd, resultados que estão de acordo com o encontrado por Hou et al., 1996.

\section{CONCLUSÕES}

1. As frações de B predominantes nos sol os são: o $B$ residual ou associado a minerais silicatados do solo (19,6 a 70,7\% do B-Total); o B associado a óxidos de Fee Al cristalinos (18,3 a 46,7\% do B-Total); o B associado a óxidos de Fe e Al amorfos $(6,4$ a $30 \%$ do B-Total) e o B associado a óxidos de $\mathrm{Mn}(0,65$ a 10,69\% do B-Total).

2. As frações de B solúvel, B não especificamente adsorvido eB especificamente adsorvido encontramse em pequenas quantidades no solo, constituindo entre 0,32 e 4,43\% do B-Total.

3. O boro solúvel em água é influenciado principalmente pelo conteúdo de matéria orgânica do solo, enquanto as frações do boro não especificamenteadsorvido e do boro especificamente adsorvido são influenciadas, além da matéria orgânica, pel os conteúdos deargila e dos óxidos ferro e alumínio do solo.

4. $\mathrm{O}$ boro extraído pela água quente, $\mathrm{HCl}$ $0,05 \mathrm{~mol} \mathrm{~L}^{-1}$ e manitol representa principalmente o boro das frações solúvel em água e não especificamente adsorvido ou trocável (fator intensidade), em menor proporção, o boro das frações especificamente adsorvido ou complexado na matéria orgânica e o ligado a óxidos de manganês (fator quantidade).

\section{LITE RATURA CITADA}

BATAGLIA, O.C. \& RAIJ , B. van. Eficiência de extratores na determinação de boro em solos. R. Bras. Ci. Solo, 14:25-31, 1990. 
BERGER, K.C. \& TRUOG, E. Boron determination in soils and plants. Ind. Eng. Chem. Anal. Ed., 11:540-544, 1939.

BIGGAR, J.W. \& FIREMAN, M. Boron adsorption and release by soils. Soil Sci. Soc. Am. Proc., 24:205-210, 1960.

BISWAPATI, M. \& KUMAR DE, D. Depthwise distribution of extractable boron in some acidic Incepptisols of India. Soil Sci., 155:256-262, 1993.

CATANI, R.A.; ALCARDE, J.C. \& KROLL, F.M. Extração e determinação do boro solúvel dos solos. An. ESALQ, Piracicaba, 27:287-294, 1970.

CARTWRIGHT, B.; TILLER, K.G.; ZARCINAS, B.A, \& SPOUNCER, L.R. The chemical assessment of boron status of soils. Aust. J . Soil Res., 21:321-332, 1983.

CHAO, T.T. Selective dissolution of manganese oxides from soils and sediments with acidified $\mathrm{NH}_{2} \mathrm{OH}$. HCl. Soil Sci. Soc. Am. Proc., 36:764-768, 1972.

CHAO, T.T. \& ZHOU, L. Extraction techniques for selective dissolution of amorphous iron oxides from soils and sediments. Soil Sci. Soc. Am. J ., 47:225-232, 1983.

CORREA, A.E.; PAVAN, M.A. \& MIYAZAWA, M. Aplicação de boro no solo e respostas do cafeeiro. Pesq. Agropec. Bras., 20:177-181, 1985.

ELSEEWI, A.A. \& ELMALKY, A.E. Boron distribution in soils and waters of Egypt. Soil Sci. Soc. Am. J ., 43:297-300, 1979.

EMPRESA BRASILEIRA DE PESQUISA AGROPECUÁRIA EMBRAPA. Manual de Métodos de Análise de Solos. 2.ed. Rio de J aneiro, 1997. 212p.

GESTRING, E.D. \& SOLTANPOUR, P.N. Evaluation of ammonium bicarbonate DTPA soil test for assessing boron availability to alfafa. Soil Sci. Soc. Am. J ., 48:96-100, 1984.

GESTRING, E.D. \& SOLTANPOUR, P.N. Comparison of soil tests for assessing boron toxicity to alfafa. Soil Sci. Soc. Am. J ., 51:1214-1219, 1987.

GOLDBERG, S. Chemistry and mineralogy of boron in soils. In: GUPTA, U.C. Boron and its role in crop production. Boca Raton, CRC Press, 1993. p.3-44.

GOLDBERG, S., Reactions of boron with soils. PI ant Soil, 193:3548, 1997

HARRISON, R.M.; LAXE, D.P.H. \& WILSON, S.J . Chemical association of lead, cadmium, copper and zinc in street dusts and roadside soils. Environ. Qual., Sci. Technol., 15:13781383, 1981.

HATCHER, J .T.; BOWER, C.A. \& CLARK, M. Adsorption of boron by soils as influenced by hydroxy aluminum and surface area. Soil Sci., 104:422-426, 1967.

HINGSTON, F.J . Reaction between boron and clays. Aust. J . Soil Res., 2:83-95, 1964.
HOU, J .; EVANS, L.J . \& SPIERS, G.A. Boron fractionation in soils. Comm. Soil Sci. Plant Anal., 25:1841-1853, 1994.

HOU, J .; EVANS, L.J . \& SPIERS, G.A. Chemical fractionation of soil boron: I. Method development. Can J . Soil Sci., 485491, 1996.

J ACKSON, M.L. Analisis químico de suelos. 2.ed. Barcelona, Omega, 1970. 662p.

J ACOMINE, P.T.K.; ALMEIDA, J.C. \& MEDEIROS, L.A.R. Levantamento Exploratório - Reconhecimento de Solos do Estado do Ceará. Recife, MA/DNPEA/SUDENE/DRN, 1973a. v.1. 301p. (Boletim Técnico, 28; Série Pedologia, 16)

J ACOMINE, P.T.K.; ALMEIDA, J.C. \& MEDEIROS, L.A.R. Levantamento Exploratório - Reconhecimento de Solos do Estado do Ceará. Recife, MA/DNPEA/SUDENE/DRN, 1973b. v.2. 502p. (Boletim Técnico, 28; Série Pedologia, 16)

J EFFREY,A.J . \& MCCALLURN, L.E. Investigation of a hot 0.01M $\mathrm{CaCl}_{2}$ soil boron extraction procedure followed by ICP-AES analysis. Comm. Soil Sci. Plant Anal., 19:663-673, 1988.

JIN-YUN J .; MARTENS, D.C. \& ZELAZNY, L.W. Distribution and plant availability of soil boron fractions. Soil Sci. Soc. Am. J ., 51:1228-1231, 1987.

JIN-YUN J .; MARTENS, D.C. \& ZELAZNY, L.W. Plant availability of applied and native boron in soils with diverse properties. Plant Soil, 105:127-132, 1988.

KEREN, R. \& BINGHAM, F.T. Boron in water, soil sand plants. In: ADVANCES IN SOIL SCIENCES. New York, SpringerVerlag Inc., New York, 1985. v.1, p..229-276.

KEREN, R. \& MEZUMAN, U. Boron adsorption by clay minerals using a phenomenological equation. Clays Clay Miner., 29:198-204, 1981.

KEREN, R.; GROSSI, P.R. \& SPARKS, D.L. Equilibrium and kinetics of borate adsorption-desorption on pyrophylite in aqueous suspensions. Soil Sci. Soc. Am. J ., 58:1116-1122, 1994.

LINDSAY, W.L. I norganic equilibria affecting micronutrients in soils. In: MORTEVEDT J .J ., COX F.R., SHUMAN, L. M. \& WELCH, R.M., eds. Micronutients in agriculture. Madison, Soil Science Society of Ammerica, 1991. p.89-112.

PARKER, D.R. \& GARDNER, E.H. The determination of hotwater soluble boron in some acid Oregon soils using a modified azomethine-H procedure. Comm. Soil Sci. Plant Anal., 12:1311-1322, 1981.

PARKS, W.L. \& WHITE, J .L. Boron retencion by clay and humus systems saturated with various cations. Soil. Sci. Soc. Am. Proc., 16:298-300, 1952.

PONNAMPERUMA, F.N.; CAYTON, M.T. \& LANTIN, R.S. Dilute hydrochloric acid as an extractant for availablezinc, copper and boron in rice soil. Plant Soil, 61:297-310, 1981. 
SHORROCKS, V.M. The occurrence and correction of boron deficiency. Plant Soil, 193:121-148, 1997

SIMS, J .R. \& BINGHAM, F.T. Retencion of boron by layer silicates, sesquioxides, and soil materials. II. Sesquioxides. Soil Sci. Soc. Am. Proc., 32:364-369, 1968.

SPOUNCER, L.R.; NABLE, R.O. \& CARTWIGHT, B. A procedure for the determination of soluble boron in soils ranging widely in boron concentrations, sodicity, and pH. Comm. Soil Sci. Plant Anal., 23:441-453, 1992.

TESSIER, A.; CAMPBELL, P.G.C. \& BISSON, M. Sequential extraction procedure for the speciation of particulate trace metals. Anal. Chem., 51:844-851, 1979.
TSADILAS, C.D.; YASSOGLOU, N.; KOSMAS, C.S. \& KALLIANOU, C.H. The availability of soil boron fractions to olive trees and barley and their relationships to soil properties. Plant Soil, 162:211-217, 1994.

WOLF, B. The determination of boron in soil extracts, plant materials, composts, water and nutrient solutions. Comm. Soil Sci. Plant Anal., 2:363-374, 1971.

WOLF, B. I mprovents in the azomethine-H method for determination of boron. Comm. Soil Sci. Plant Anal., 5:3944, 1974.

YERMIYAHO, U.; KEREN, R. \& CHEN, Y. Boron sorption on composted organic matter. Soil Sci. Soc. Am. J ., 52:1309, 1988. 